\title{
Ovarian Cancer: Role of Cytology
}

\author{
SANDEEP R MATHUR
}

Cytology has a limited role to play in the primary diagnosis of ovarian cancer except when it presents with malignant ascitis in the patient. Frequently detection of malignant ascitis is the first diagnostic evidence which leads to other modalities of investigations. Accuracy of imaging techniques like ultrasound \& CT scan in detecting malignancy and omental or peritoneal deposits, large variety of tumours difficult to subtype on cytology alone, fear of spillage of tumour cells into the peritoneal cavity, inexperience of cytopathologists in interpreting ovarian lesions have been the reasons why most clinicians not resorting to fine needle aspiration of ovarian mass.

However there are certain situations where cytology both FNA as well as exfoliative have an important role to play:

1) Ascitic or pleural fluid cytology for detection of malignant cells

2) Peritoneal washings at the time of surgery to detect peritoneal deposits

3) In detecting suspected pelvic recurrences in known cases of ovarian cancers

4) In arriving at a primary diagnosis of ovarian cancer in patients who are poor surgical risk

5) In distinguishing non neoplastic cyst from true neoplasms of the ovary

6) Patients already having a disseminated disease at presentation

Department of Pathology, AIIMS, New Delhi-110029 mathuraiims@yahoo.com
7) In detected metastatic tumour deposits at unusual sites like cutaneous location in known cases of ovarian cancers

\section{Technique:}

Almost always performed under radiologic guidance, preferably ultrasound. Transvaginal route is a favoured approach. Transabdominal, transrectal, laparoscopic and aspiration at the time of laparotomy are some of the other approaches possible. Transabdominal approach is suited for large masses with omental deposits.

\section{Sample preparation and processing:}

It is advisable to have at least two alcohol-fixed and air-dried smears stained with Papanicolaou and May Grunwald Giemsa stains respectively. The radiologist performing the procedure should be particularly careful to avoid air drying artifacts and ensure even spreading of aspirated material. Extra unstained smears should be prepared for immunocytochemistry.

\section{Clinical and radiologic details}

They are crucial adjuncts in arriving at a correct diagnosis. This may include information about approach for aspiration, presence of ascitis, hormonal profile, CA-125 levels, USG, CT scan findings, tumour markers like alpha-feto protein (AFP), placental alkaline phosphatase (PLAP), beta HCG, etc.

\section{Scope of Cytomorphology in diagnosis of common malignant ovarian neoplasms}

\section{Borderline tumours of ovary}

Borderline serous \& mucinous neoplasms are difficult to differentiate from malignant 
counterpart as invasion cannot be documented on cytology. They usually present as highly cellular smears with nuclear features ranging from bland to highly atypical. A definitive diagnosis of a borderline ovarian surface epithelial tumour is not possible on cytologic material

\section{Malignant tumours of ovary}

The cytologic diagnostic accuracy for ovarian carcinoma is in the range of $90-95 \%$. A negative diagnosis in a suspected case does not rule out malignancy and should warrant a repeat procedure. Subtyping is relatively easier in the low and intermediate grade tumours, but is not possible in the high grade varieties. The germ cell tumours (GCT) constitute the majority of ovarian masses in the pediatric age group. Most GCTs can be reliably diagnosed and subtyped on FNAC, in conjunction with tumour marker levels and immunocytochemistry. Endometrioid tumours display features like those of the endometrial counterpart, but may be difficult to distinguish from serous cytadenocarcinomas. Malignant mixed mullerian tumours can only be suspected when both epithelial and sarcomatous component are aspirated, otherwise it may be classified as an adenocarcinoma. Granulosa cell tumour can be diagnosed on FNA in the presence of acinar like structures with central reddishviolet bodies called as Call-exner bodies and cells with nuclear grooves. Aspirates from stromal tumours of ovary may yield very sparse material and may be difficult to diagnose on FNAC.

\section{Role of immunocytochemistry}

Most of the surface epithelial malignancies can be diagnosed without resort to immunocytochemistry. Immunocytochemistry for inhibin in conjunction with Ber EP4, CK 7 and CA-125 may help in differentiating follicular cells in non-neoplastic cyst aspirates from epithelial proliferations in neoplastic masses. Metastatic tumours may also be distinguished from primary using an immunocytochemistry panel. Antibodies against PLAP, AFP and beta HCG may help in classifying germ cell tumours. 